Lexis Vol. XLIV (2) 2020: 805-822

\title{
El cronotopo en la space opera: un análisis comparativo con la novela de aventuras
}

\author{
Jose Luis Arroyo Barrigüete \\ Universidad Pontificia Comillas
}

\section{RESUMEN}

En este trabajo analizamos las características propias de la novela de aventuras y pruebas que Bajtín identifica como uno de los tres tipos esenciales de unidad novelesca. La exégesis de este cronotopo nos revela un esquema muy similar al que aparece en un popular subgénero de la ciencia ficción, esto es, la space opera. Nuestra conclusión es que ambos cronotopos, el de aventuras y pruebas, y el de la space opera, son bastante similares, salvo por un aspecto muy relevante: la reversibilidad temporal, ausente en este último como consecuencia de un menor protagonismo del suceso. Esto es consecuencia de una axiología heroica más propia de lo épico que de la novela, aspecto que también se analiza en el trabajo.

Palabras clave: cronotopo, Bajtín, space opera, novela de aventuras y pruebas

The Chronotope in Space Opera: A Comparative Analysis with the Adventure Novel

\section{Abstract}

In this paper we analyze the characteristics of the adventure novel of ordeal, which Bakhtin identifies as one of the three essential types of novelistic

https://doi.org/10.18800/lexis.202002.012 
unity. The exegesis of such chronotope reveals a pattern not unlike that which articulates a popular sub-genre of science fiction: space opera. Our conclusion is that both chronotopes are virtually identical except for a very relevant aspect, temporal reversibility, absent in space opera, because of a lesser protagonism of the event. This is the result of a heroic axiology that is more typical of the epic than of the novel, an aspect that is also analyzed in the paper.

Keywords: chronotope, Bakhtin, space opera, adventure novel

\section{Introducción}

Bajtín ([1924] 1989: 237) define el cronotopo como "la conexión esencial de relaciones temporales y espaciales asimiladas artísticamente en la literatura”, es decir, sería el tratamiento del espacio y el tiempo en la obra literaria, aunque considerando ambos aspectos conjuntamente y poniendo el foco en la relación que existe entre ambos. Este último aspecto, la relación entre el espacio y el tiempo, es precisamente la clave del concepto Bajtiano de cronotopo, puesto que su teoría considera imprescindible el estudio conjunto. Aunque sea anticiparnos a algo que desarrollaremos más adelante, amerita ejemplificar este punto con el caso de la novela de aventuras y pruebas. Estas novelas habitualmente se desarrollan en un espacio amplio, cuyos protagonistas han de recorrer superando diversas dificultades. Esto requiere, entre otras cosas, largos periodos de viaje, es decir, mucho tiempo. En el mundo ordinario, implicaría que los personajes envejezcan, lo que sería un problema a efectos narrativos, y esta es la razón por la que en estas novelas existe un hiato extratemporal en el tiempo biográfico, de modo que el tiempo empleado en cada una de las aventuras y viajes no se suma, y, por tanto, no deja huella en los actantes.

Esta definición del cronotopo, aparentemente sencilla, contiene tal cantidad de sutilezas que el autor se vio en la necesidad de desarrollar su significado en un extenso ensayo (237-409) cuyas 
conclusiones aún siguen vigentes en la actualidad ${ }^{1}$. Concretamente, Bajtín identifica tres tipos esenciales de unidad novelesca, y, por lo tanto, tres cronotopos novelescos (239). El primero de ellos, que es, además, el que resulta de interés para el presente trabajo, es el de la novela de aventuras y pruebas, que incluye a la "novela griega" o "sofística", en las que "la elaboración de este tiempo de la aventura y la técnica de utilización del mismo en la novela son tan perfectos ${ }^{2}$ que la evolución posterior de la novela pura de aventuras no ha añadido nada importante hasta nuestros días” (240). En el segundo tipo, la novela de aventuras costumbrista (263-282), caracterizada por la combinación del tiempo de la aventura y el de las costumbres, encontramos que el camino de la vida del héroe, ligado al viaje en la novela, se representa como una metamorfosis. Por último, la novela biográfica (282-298) proyecta una imagen del hombre que "recorre su camino de la vida” (283).

El enfoque propuesto por Bajtín, esto es, el análisis de las relaciones espaciales y temporales como un todo vinculado y no como elementos independientes, ha dado lugar a investigaciones muy provechosas en el ámbito de la literatura, incluyendo la ciencia ficción ${ }^{3}$. En este sentido resulta interesante cómo Suvin, uno de los mayores especialistas en este género, modifica su definición de ciencia ficción. En uno de sus trabajos más icónicos, el libro Metamorphoses of Science Fiction, definía el género como “a literary genre whose necessary and sufficient conditions are the presence and interaction of estrangement and cognition, and whose main formal device is an

\footnotetext{
Aun así, como señalan Bemong y Borghart (2010: 5), "lack of analytical precision in Bakhtin's essays has led to a proliferation of heterogeneous chronotopic approaches to literature and, more generally, culture". Estos investigadores definen cinco niveles de abstracción diferentes (6-8), y en nuestro caso nos centraremos en lo que denominan generic chronotopes, esto es, aquellos que dan lugar a un determinado género literario.

2 Bajtin se refiere al hecho de que el tiempo de la aventura en este tipo de novelas es elaborado "cuidadosa y sutilmente, con todas sus características y matices específicos"(240). 3 Empleamos aquí el término ciencia ficción en su sentido más amplio. Moreno (2013) propone diferenciar entre literatura de ciencia ficción y literatura prospectiva según su efecto, de asombro en el primer caso, de replanteamiento de cuestiones socio-culturales en el segundo. Aun estando de acuerdo con esta diferenciación, en este trabajo emplearemos el término ciencia ficción como globalizador de ambas tipologías.
} 
imaginative framework alternative to the author's empirical environment" ([1979] 2016: 20) [las cursivas son nuestras]. En trabajos más recientes, modifica esta definición para incorporar, precisamente, el concepto de cronotopo: “a literary (etc.) genre defined by the interaction of estrangement and historical cognition, and whose main formal device is a narrative chronotope and/or agents alternative to the author's empirical world" (2008: 116) [Las cursivas son nuestras].

No es de extrañar, por tanto, que diversos académicos hayan empleado el cronotopo como elemento de análisis en sus investigaciones sobre el género de ciencia ficción. Por citar algunos trabajos recientes, Clark, en su tesis doctoral, analiza el cronotopo en la ciencia ficción, afirmando que "[i]f genres actually represent different kinds of spatio-temporal narrative worlds, then science fiction, like all other genres, ought to have its own distinctive kind of time and space" (2017: ii). Skult (2012), tomando como base el cronotopo del encuentro y el cronotopo de la novela de viaje, propone un cronotopo propio para la novela de ciencia ficción postapocalíptica ${ }^{4}$, análisis que es luego aprovechado en gran medida por Ciftcibasi (2016) cuando estudia la novela The Drought (1965) de J. G. Ballard. Gomel (2009) analiza el cronotopo en la novela de viajes en el tiempo, mientras que Pak (2016) lo estudia en novelas que incluyen el proceso de terraformación de planetas. En la línea de los trabajos mencionados, la pregunta que nos hacemos en este artículo es, precisamente, cuáles son las características del cronotopo en uno de los subgéneros de ciencia ficción más populares, esto es, la space opera.

Según señala Pringle (2000: 35), el término space opera fue acuñado por el escritor estadounidense Wilson Tucker en 1941 para referirse a "hacky, grinding, stinking, outworn, spaceship yarn", aunque actualmente se han perdido gran parte de estas connota-

\footnotetext{
4 Sin embargo, Pisarska (2015) afirma que el cronotopo post apocalíptico de la novela The Pesthouse (2007), de J. Crace, surge por la interacción de los cronotopos genéricos utópico y distópico.
} 
ciones negativas. Wolfe (1986: 120), en su glosario de términos de la ciencia ficción, señala que "space operas are generally fast-paced intergalactic adventures on a grand scale”; Moreno (2013) apunta que "serían los equivalentes espaciales a los cantares épicos medievales o a los libros de caballerías renacentistas, contemplados desde el punto de vista de las líneas argumentales"; y Suvin ([1979] 2016: 99) señala que "[a]ll space operas can be translated back into the Social Darwinism of the Westerns and similar adventure-tales by substituting colts for ray-guns and Indians for the slimy monsters of Betelgeuse".

En nuestra opinión, una definición bastante completa de lo que significa la space opera es la que propone Pringle:

Put together the following ingredients: a pinch of serious, which is to say utopian, interplanetary sf; a large dollop of planetary romance; an equally large dollop of the future war tale, or Edisonade; and a bucketful of salty sea yarn; and, voila, you have the makings of space opera. But in fact this leaves out at least one more very important ingredient: what could be termed "cosmicism". Since it is set on spaceships, between planets, space opera is clearly interplanetary, interstellar or even intergalactic fiction (2000: 41).

Académicos como Peregrina Castaños (2014: 159, 209-210) apuntan la similitud de su cronotopo con el de la novela de aventuras, algo que, sin embargo, en nuestra opinión no ha sido suficientemente estudiado. Coincidimos plenamente con Peregrina Castaños en que el cronotopo de la space opera comparte elementos con la novela de aventuras, y aun así consideramos necesario realizar una serie de matizaciones relevantes. A lo largo de este trabajo, analizaremos en profundidad las características del cronotopo en ambos géneros, tratando de probar que existen algunas diferencias importantes, en especial en lo que se refiere a su dimensión temporal. 


\section{El tiempo y el espacio}

Según Bajtín, el cronotopo en la novela de aventuras y pruebas se caracteriza por "la ligazón técnica abstracta entre el espacio y el tiempo, por la reversibilidad de los momentos de la serie temporal y por la transmutabilidad del mismo en espacio" (253). Esta definición, notablemente compleja, requiere de una mayor explicación, por lo que profundizaremos en el significado de cada uno de sus elementos. En primer lugar, resulta interesante ahondar en las ideas de ligazón y transmutabilidad, es decir, en cómo el tiempo se configura espacialmente, y el espacio adquiere una dimensión temporal. En la novela de aventuras y pruebas, ambos elementos quedan íntimamente relacionados, de modo que no es posible comprender el uno sin el otro. El espacio amplio en el que se suelen desarrollar las aventuras requiere necesariamente de un tiempo de la novela desligado del tiempo biológico de los personajes, pues solo de este modo pueden compatibilizarse largos viajes con un imperceptible envejecimiento de los actantes. Del mismo modo, la ausencia de tiempo histórico necesita de un espacio abstracto, dando lugar de manera conjunta a lo que Bajtin denomina transferibilidad temporal. Profundicemos en esta ligazón analizando detenidamente las distintas características del tiempo, y el espacio en la novela de aventuras y pruebas y en la space opera.

Bajtín señala que, en la novela de aventuras y pruebas, el tiempo no deja huella alguna en los personajes (243), pues existe un "hiato extratemporal entre los dos momentos del tiempo biográfico” (242), ya que el tiempo empleado en cada una de las aventuras no se suma en una serie temporal (247). Del mismo modo, el tiempo tampoco deja huella alguna en el mundo (244), pues "el universo y el hombre aparecen como productos totalmente acabados e inmutables [...], nada en este universo es destruido, rehecho, modificado, creado de nuevo" (263). Esta ausencia de huella temporal es lo que Bajtín denomina "reversibilidad". Adicionalmente, la tercera caracterís-

5 “el tiempo en la novela no deja ningún tipo importante de huella y es en esencia, por tanto, reversible” (253). 
tica de este tipo de novela es la ausencia de "tiempo histórico” (244), es decir, la inexistencia de indicios de la época, lo que nos lleva a la transferibilidad temporal de la obra.

En relación con la primera característica que menciona Bajtín, efectivamente, y como norma general, los personajes de la space opera no parecen demasiado afectados por el tiempo biológico. Los protagonistas son capaces de increíbles hazañas, en muchas ocasiones de tipo físico, sin que el tiempo parezca mermar sus capacidades. Compatibilizar este hecho con los viajes interplanetarios a enormes distancias requiere, forzosamente, de los viajes instantáneos, de modo que un elemento clave en el pacto ficcional ${ }^{6}$ es, precisamente, este tipo de viaje. Hasta donde sabemos, los viajes a velocidades supralumínicas son una imposibilidad física, de manera que, para evitar incurrir en contradicciones científicas, suele recurrirse a conceptos como el hiperespacio, los agujeros de gusano o el salto a través de dimensiones superiores. Algunas novelas exploran, sin embargo, los efectos relativistas ${ }^{7}$ del viaje interestelar en un universo en el que no existe la posibilidad de los viajes instantáneos, siendo dos de los ejemplos más conocidos The Forever War (1974) de Joe Haldeman, y Speaker for the Dead (1986) de Orson Scott Card.

Por tanto, la ciencia y tecnología juegan un papel esencial en el cronotopo de la space opera, pues, como acabamos de analizar, proporcionan las herramientas ficcionales necesarias para hacer plausible ese "hiato extratemporal" sin incurrir en contradicciones científicas, algo crítico en el ámbito de la ciencia ficción dura. Como señala Csicsery-Ronay Jr. (2008: 220), “SF’s imaginary science, often

\footnotetext{
6 Moreno (2013: pos. 1610) define el "pacto de ficción” como "el acuerdo establecido entre un texto ficcional y un lector de dicho texto, por el cual ese lector lee los sucesos ocurridos como si estuvieran de verdad ocurriendo ante él".

7 Nos referimos concretamente al hecho de que el tiempo transcurre más lentamente a medida que aumenta la velocidad, de modo que un viajero espacial, moviéndose a una fracción significativa de la velocidad de la luz, percibirá un tiempo de días o semanas, mientras en el resto del universo han transcurrido años o incluso siglos. Este es uno de los resultados que se deriva de la teoría de la relatividad especial de Einstein, cuyo artículo seminal fue publicado en 1905 en la revista "Annalen der Physik".
} 
based $[\ldots]$ on scientific speculations that cannot be demonstrated actually to be impossible, provides the nondialectical chronotopes of adventure with a powerful, quasi-scientific nibil obstat".

La tercera característica que señala Bajtín, la ausencia de tiempo histórico, también está presente en la space opera, aunque en este caso es una consecuencia de la ambientación futurista. Una vez que nos movemos mil años en el futuro, tanto da que sean dos mil o diez mil, ya que carecemos de referentes que nos permitan ubicar la acción en un determinado contexto histórico; es decir, la ambientación futurista y la ausencia de tiempo histórico presentan una relación casi tautológica.

Sin embargo, en lo que se refiere a la ausencia de huella del tiempo en el universo, encontramos una diferencia sustancial entre la space opera y la novela de aventuras, pues la esencia de la primera es, precisamente, que la actuación del héroe sí tiene un impacto mensurable y significativo en la sociedad. Aunque no pertenece al ámbito de la literatura, la space opera por antonomasia es la saga cinematográfica de Star Wars, en donde la actuación de los héroes reconfigura por completo el sistema político de la galaxia, derrocando al Imperio y abriendo la puerta a una república democrática. Resulta difícil imaginar un cambio de mayor magnitud. En la trilogía Foundation (1951-1953) ${ }^{8}$, de Isaac Asimov, un científico altera por completo el curso de la galaxia, acelerando la aparición de un Segundo Imperio para reemplazar al decadente Primer Imperio; y en Dune (1965-1985), de Frank Herbert, otra de las sagas míticas, al igual que sucede en Star Wars, el protagonista cambia completamente el sistema político y los equilibrios de poder en la galaxia. Son solo tres ejemplos de que la naturaleza de la space opera demanda que la actuación del héroe tenga un impacto significativo en su universo, negando por tanto esa reversibilidad del tiempo a la que Bajtín hace referencia.

\footnotetext{
8 Nos referimos a la trilogía nuclear, compuesta por Foundation, Foundation and Empire y Second Foundation. La meta saga completa está formada por un conjunto de quince novelas y varias de ellas no entrarían dentro de la categoría de space opera.
} 
En resumen, al igual que en la novela de aventuras y pruebas, en la space opera, habitualmente aparece un "hiato extratemporal entre los dos momentos del tiempo biográfico", que se compatibiliza con las distancias inconcebiblemente grandes mediante la introducción del viaje instantáneo. Es por esta razón que el aparato tecnológico juega un papel clave en la configuración del cronotopo en la space opera, permitiendo dicha compatibilidad de un modo creíble. Del mismo modo, también se pone de manifiesto una ausencia de tiempo histórico, que realmente es consecuencia de la ambientación futurista. Sin embargo, en el caso de la space opera, el tiempo no es reversible, pues la actuación de los protagonistas sí deja huella en "su" mundo. He aquí la primera diferencia significativa entre ambos cronotopos.

Por otra parte, Bajtín señala que el tiempo descrito hasta este momento, y que caracteriza a la novela de aventuras y pruebas, necesita de una extensión espacial amplia y abstracta (252), afirmación que clarifica de un modo muy gráfico cuando señala que " $[\mathrm{p}]$ ara el naufragio se necesita el mar, pero no tiene importancia qué mar sea ese desde el punto de vista geográfico e histórico" (252). Esto nos lleva a la transferibilidad, pues "lo que sucede en Babilonia, podría suceder en Egipto o en Bizancio y viceversa” (253), lo que requiere, entre otras cosas, un protagonista "aislado, carente por completo de relaciones, más o menos importantes, con su ciudad, con su grupo social, con su clan, e incluso con su familia. No se considera parte del conjunto social" (261). Una segunda característica del espacio es su carácter ajeno, lleno de cosas "inacostumbradas, extrañas, raras” (255). Según Bajtín, esto es un imperativo dado el protagonismo del suceso, del que hablaremos en el siguiente apartado, pues toda concreción geográfica, económica o de otro tipo, que sería inevitable en el caso de la representación de la realidad, limitaría el poder absoluto del suceso (253). En la novela de aventuras y pruebas, muchos fenómenos están descritos en detalle, lo que parece incompatible con ese carácter abstracto que mencionábamos antes. Bajtín aclara que estas descripciones son aisladas, pues “[e]n ninguna parte se describe el País en su totalidad [...]. Sólo 
se describen ciertos edificios sin ninguna relación con el conjunto integrador [...]. En ninguna parte se describen las costumbres [...] en su conjunto; únicamente se describe alguna costumbre rara, sin ninguna relación con otras cosas. Todos los objetos descritos en la novela se caracterizan por ese aislamiento, esa falta de relación entre sí" (254-255). De hecho, en estas novelas abundan las cosas "curiosas y llenas de extrañezas" (255).

En el caso de la space opera, su propia definición implica un espacio amplio, abstracto y transferible. En la medida en que la acción transcurre en distintos planetas, obviamente incorporamos la vastedad cósmica al relato; y, en tanto que los planetas son, forzosamente, inventados, tanto da el nombre o características que les asignemos, pues su única función en el relato es servir a los propósitos narrativos 9 . Volviendo a la frase de Bajtín, "para el naufragio se necesita el mar, pero no tiene importancia qué mar sea ese desde el punto de vista geográfico e histórico"; de igual modo, para una aventura submarina, se necesita un planeta acuático, pero no tiene importancia cuál sea este, de modo que, a efectos narrativos, tanto nos da si se trata de Europa, el satélite joviano o de un planeta completamente inventado en la órbita de Tau Ceti ${ }^{10}$.

En esta misma línea, el hecho de que la ambientación esté llena de cosas “inacostumbradas, extrañas, raras” es también una consecuencia derivada de la propia definición de space opera. De manera que, efectivamente, este subgénero parece seguir, punto por punto, la descripción dada por Bajtín. No insistiremos más en este particular, pues, como hemos visto, nos hallamos de nuevo ante una tautología: la propia definición de space opera exige el espacio Bajtiano de la novela de aventuras y pruebas.

\footnotetext{
9 Cabe añadir el importante matiz de la plausibilidad científica. Si hoy escribiésemos una novela de ciencia ficción ambientada en el planeta Mercurio, la descripción debería coincidir con lo que sabemos de este planeta o dar una explicación plausible de las diferencias, en el caso, por ejemplo, de que haya sido sometido a terraformación.

10 Una estrella relativamente cercana al sistema solar, aproximadamente doce años luz, que resulta interesante para la comunidad científica por sus características similares a las de nuestro Sol.
} 


\section{El protagonismo del suceso}

Bajtín apunta, en relación con la novela de aventuras y pruebas, que "[e]n este cronotopo, la iniciativa y el poder pertenecen únicamente al suceso" (253), pues habitualmente se organizan como pruebas del héroe y de la heroína, en las que se evalúa "su nobleza, su valentía, su fuerza, su intrepidez, y — más raramente— su inteligencia” (259). De hecho, señala que "el hombre sólo puede ser totalmente pasivo e inmutable [...] al hombre le sucede todo. Él mismo carece por completo de iniciativa. Es sólo el sujeto físico de la acción” (258). En la space opera esto es, en general, bastante cierto, pues habitualmente es un determinado suceso el que desencadena el resto de acontecimientos, que los protagonistas tratan de ir solventando a medida que se suceden. Sagas ampliamente conocidas como Dune de Frank Herbert; la trilogía Foundation de Asimov; la saga de Ender de Orson Scott Card; y, más recientemente, Ringworld de Larry Niven o la saga de Miles Vorkosigan de Lois McMaster Bujold son algunos ejemplos de este "protagonismo del suceso". En el caso de Dune, la traición y asesinato del duque Leto Atreides obliga a su hijo, Paul Atreides, a exiliarse en el desierto, lo que, tras una serie de duras pruebas, lo lleva a convertirse en el Kwisatz Haderach, líder de la tribu de los Fremen. En la trilogía Foundation, la inminente caída del Imperio Galáctico lleva a Hari Seldon, un brillante científico, a crear dos fundaciones custodias del conocimiento, a fin de disminuir el periodo de barbarie que sobrevendrá tras la caída y hasta la formación de un Segundo Imperio. Las tres novelas, de hecho, tienen como hilo conductor la superación de diversas crisis por parte de la Fundación, que sobrevienen, una tras otra, sin que pueda hacerse nada para evitarlo ${ }^{11}$. En la Saga de Ender, un joven niño prodigio es obligado a entrenarse como militar a fin de liderar

\footnotetext{
11 Esto suele considerarse como una clara influencia del historiador Arnold J. Toynbee, quien opinaba que las civilizaciones crecen y progresan a medida que se enfrentan a desafíos que solucionan satisfactoriamente: sin desafíos la civilización decae, y, ante desafíos que no puede resolver, colapsa. De hecho, la influencia de Toynbee en la trilogía es algo que mencionan diversos académicos como Hassler (1988), Irwin (1997), White (2005: 85), Sawyer (2009: 491) o Seed (2011: 100), entre otros.
} 
el ataque contra una raza extraterrestre. Su duro entrenamiento, con infinidad de obstáculos, conforma la mayor parte del primer libro. Las consecuencias de su actuación en el mismo devienen en otra serie de problemas que trata de solucionar en las novelas posteriores. En Ringworld, un extravagante equipo formado tanto por humanos como por extraterrestres, explora un misterioso mundo construido por una civilización desconocida, en donde, una tras otra, se enfrentan a pruebas y dificultades. En la primera novela de la saga ${ }^{12}$ de Miles Vorkosigan, un joven de noble linaje, brillante, pero con deformidades físicas, tras ser rechazado en la Academia Imperial, y como consecuencia de una serie de acontecimientos fortuitos, acaba creando su propia flota mercenaria. La estructura del relato no deja de ser curiosa, pues, tratando de solucionar cada dificultad sobrevenida, Miles genera un problema aun mayor, en una frenética huida hacia adelante. En novelas posteriores de la saga también se repite el patrón de sucesos que afectan al protagonista, sin que él los haya buscado de manera consciente.

Sin embargo, hay un matiz importante, pues el sujeto no carece por completo de iniciativa, ya que, si bien suele verse sumido en una serie de pruebas repentinas e imprevistas, es muy habitual que en algún momento el protagonista adopte un papel activo, tomando la iniciativa precisamente para evitar convertirse en una simple marioneta del destino. Este matiz enlaza con lo que mencionábamos en el segundo apartado, al reflexionar sobre la naturaleza del tiempo en la space opera, pues es al recuperar la iniciativa cuando el héroe es capaz de producir una huella en su universo, eliminando la reversibilidad temporal. Este es, en nuestra opinión, el elemento clave que diferencia el cronotopo en la space opera respecto a la novela de aventuras y pruebas que describe Bajtín.

\footnotetext{
12 Nos referimos a la novela The Warrior's Apprentice (1986), la primera en la que aparece Miles Vorkosigan. Realmente no es la primera novela de la saga, pues suele considerarse que la saga incluye, al menos, tres novelas anteriores al nacimiento de Miles.
} 


\section{Lo fronterizo y lo híbrido en el cronotopo de la space opera}

Llegados a este punto, parece pertinente analizar en qué medida el cronotopo de la space opera, lejos de configurarse como una categoría estanca, comparte elementos con otros géneros más allá de la novela de aventuras y pruebas, centrándonos concretamente en el cronotopo épico ${ }^{13}$. Como Bajtin señala, "the epic hero lacks any ideological initiative [...]. The epic world knows only a single and unified world view, obligatory and indubitably true for heroes as well as for authors and audiences" ([1941] 1996: 56). Esta visión, un tanto maniquea, es una característica que efectivamente encontramos en la space opera, generando ciertas resonancias del prototipo épico al que Bajtin hace referencia. Del mismo modo, "[...] epic concerns itself with glorious deeds and heroic destiny [...]" (Falconer 2010: 113), una concepción que coincide plenamente con la propia de la space opera; es decir, parece que la space opera comparte estos elementos con la axiología mítica y heroica propia de lo épico.

Por otra parte, la separación absoluta del universo ficcional con respecto a la realidad actual es otro elemento también propio de la épica que también encontramos en la space opera: "an absolute epic distance separates the epic world from contemporary reality, that is, from the time in which the singer (the author and his audience) live" (Bajtin ([1941] 1996: 52). La diferencia se encuentra en que, en el caso de la space opera, la orientación es hacia el futuro, en vez de al pasado, aunque en esencia estamos ante una estructura idéntica, en la que existe una enorme distancia entre el mundo ficcional y la realidad del autor y el lector. De hecho, el relato épico "acentúa [...] la inaccesibilidad de su héroe, tan admirable como inimitable, precisamente porque su universo nada tiene en común con el mundo de la experiencia cotidiana [...]" (Gómez-Moriana, 1997: 167). Y, de nuevo, aquí encontramos importantes paralelismos con la space opera.

13 Hemos de indicar que Bajtin mantenía una postura bastante crítica con respecto a lo épico (Falconer 1997: 254). 
Sin embargo, también existen diferencias, pues frente al héroe inmutable y que no parece evolucionar, propio de la épica (Bajtin ([1941] 1996: 50), el héroe de la space opera sí evoluciona a medida que supera los distintos obstáculos, al menos una vez que este subgénero maduró. En sus orígenes, tal y como se ha comentado en la introducción, la escasa calidad literaria de la space opera se manifestaba en esquemas simplistas con personajes planos. Esto fue cambiando, y las obras icónicas, entre las que se encuentran varias de las mencionadas en este trabajo, no presentan, en general, esta peculiaridad. Bien es cierto que, en ocasiones, autores como Asimov tienden a priorizar el relato, relegando a un segundo plano la caracterización de los personajes, así como su evolución, pero en ningún caso puede afirmarse que esta sea una característica general de la space opera.

Como conclusión de estas reflexiones, parece que las fronteras no resultan nítidas, en tanto que en la space opera encontramos también elementos propios no solo de la novela de aventuras y pruebas, sino también de lo épico, especialmente en lo concerniente a la axiología heroica. Parecería que este subgénero se configura de algún modo como un espacio frontera entre ambos, una suerte de híbrido entre los dos géneros y cuyo cronotopo es muy similar al de la novela de aventuras y pruebas, salvo en lo que se refiere a la reversibilidad temporal, precisamente como consecuencia de una axiología heroica más propia de lo épico.

\section{Conclusiones}

Como hemos tratado de probar a lo largo de este trabajo, aunque efectivamente el cronotopo de la space opera comparte elementos con la novela de aventuras y pruebas que tan detenidamente analiza Bajtín, existe, sin embargo, una diferencia relevante. En ambos casos nos encontramos con una ausencia de huella histórica y un tiempo que no se suma en una serie temporal, generándose un hiato extratemporal en el tiempo biográfico. También, en ambos casos, estamos ante un espacio amplio, abstracto, transferible y lleno de 
cosas ajenas a nuestra experiencia, y, en el caso de la space opera, los viajes interestelares instantáneos son el elemento, dentro del contrato ficcional, que hacen compatibles el espacio y tiempo así definidos; es decir, la tecnología juega un papel clave en la configuración del cronotopo, pues permite compatibilizar de un modo plausible un tiempo "que no se suma en una serie temporal” (Bajtín ([1924] 1989: 247), con una extensión espacial amplia y abstracta (252). El aparato tecnológico es, por tanto, la pieza que permite dotar de credibilidad a este cronotopo. Esta plausibilidad, que no parece ser demasiado relevante en la novela de aventuras y pruebas, sí resulta absolutamente esencial en la space opera, al igual que en el resto de subgéneros dentro del ámbito de la ciencia ficción dura.

Hasta aquí, podemos concluir que ambos cronotopos son muy similares. Sin embargo, el protagonismo del suceso en la space opera no es tan intenso como en la novela de aventuras y pruebas, permitiendo a los protagonistas adoptar un papel activo en la trama, lo que deviene en la posibilidad de generar una huella visible y significativa en su universo, eliminando así la reversibilidad temporal. Como ya hemos apuntado, nuestra hipótesis es que esto es consecuencia de una axiología heroica más cercana a lo épico que a la novela.

Como conclusión, este trabajo realiza dos aportes diferentes. En primer lugar, un análisis pormenorizado del cronotopo de la space opera, ligándolo a elementos muy característicos de su contrato ficcional, y probando, así, que comparte ciertos aspectos con el de la novela de aventuras y pruebas. Este tipo de análisis, si bien se ha desarrollado para otros subgéneros de la ciencia ficción, como hemos comentado a lo largo del artículo, en nuestra opinión no había sido estudiado en suficiente profundidad para el caso de la space opera. El segundo aporte es identificar la diferencia clave entre ambos cronotopos, esto es, la reversibilidad temporal, pues la inmutabilidad del universo, algo habitualmente cierto en la novela de aventuras, no suele cumplirse en la space opera, como consecuencia de un menor protagonismo del suceso. 


\section{Referencias bibliográficas}

BAJTin, Mijaíl

[1924] 1989 Teoría y estética de la novela. Trad., H. Kriukova y V. Cazcarra. Madrid: Tauros.

Bajtin, Mijaíl

[1941] 1996 "Epic and Novel”. En Essentials of the Theory of Fiction. Eds., Michael J. Hoffman y Patrick D. Murphy. Londres: Leicester University Press.

Bemong, Nele y Borghart, Pieter

2013 "Bakhtin's Theory of the Literary Chronotope: Reflections, Applications, Perspectives". En Bakbtin's Theory of the Literary Chronotope: Reflections, Applications, Perspectives. Eds., Nele Bemong, Pieter Borghart, Michel De Dobbeleer, Kristoffel Demoen, Koen De Temmerman y Bart Keunen. Gent: Academia Press, 3-16.

Ciftcibasi, Ayse

2016 "Post-Apocalyptic Chronotope in J. G. Ballard's The Drought". The Journal of International Social Research. 9. 46, 79-85. <https://doi.org/10.17719/jisr.20164622577>

CSICSERY-RONAY Jr, Istvan

2008 The seven beauties of science fiction. Middletown: Wesleyan University Press. Edición de Kindle.

Clark, Andrew

2017 Reading Space-time: A Chronotopic Analysis of the Science Fiction Genre. Tesis Doctoral. Universidad de Auckland.

FALCONER, Rachel

1997 "Bakhtin and the epic chronotope". En Face to Face: Bakbtin in Russia and the West. Eds., Carol Adlam, Rachel Falconer, Vitalii Makhlin y Alastair Renfrew. Sheffield: Sheffield Academy Press, 254-272.

FALCONER, Rachel

2010 "Heterochronic Representations of the Fall: Bakhtin, Milton, DeLillo". En Bakbtin's Theory of the Literary Chronotope: Reflections, Applications, Perspectives. Eds., Nele Bemong, Pieter Borghart, Michel De Dobbeleer, Kristoffel Demoen, 
Koen De Temmerman y Bart Keunen. Gent: Academia Press, 111-130.

Gomel, Elana

2009 "Shapes of the Past and the Future: Darwin and the Narratology of Time Travel”. Narrative. 17. 3, 334-352. $<$ https://doi.org/10.1353/nar.0.0029>

Gómez-Moriana, Antonio

1997 "Triple dimensionalidad del cronotopo bajtiniano: diacronía, diatopía, diastratía”. Acta poética. 18. 1-2, 153-188.

HassLer, Donald M.

1988 "Some Asimov Resonances from the Enlightenment". Science Fiction Studies. 15. 1, 36-47.

IRWIN, Robert

1997 “Toynbee and Ibn Khaldun”. Middle Eastern Studies. 33. 3, 461-479. <https://doi.org/10.1080/00263209708701164>

Moreno, Fernando Ángel

2013 Teoría de la literatura de ciencia ficción (Spanish Edition). España: Sportula Ediciones. Edición de Kindle.

PAK, Chris.

2016 Terraforming. Ecopolitical Transformations and environmentalism in Science fiction. Liverpool: Liverpool University Press.

Peregrina Castaños, Miquel

2014 El cuento español de ciencia ficción (1968-1983): los años de 'Nueva Dimensión'. Tesis Doctoral. Universidad Complutense de Madrid.

PISARSKA, Katarzyna

2015 "America of Future Past: The Post-Apocalyptic Chronotope of Jim Crace's The Pesthouse”. Roczniki Humanistyczne. LXIII. 11, 275-290.

Pringle, David

"What is this Thing called Space Opera?" En Space and Beyond: The Frontier Theme in Science Fiction. Ed., Gary Westfahl. Westport: Greenwood Press, 35-47. 
SAWYER, Andy

2009 "Future History". En The Routledge Companion to Science Fiction. Ed., Mark Bould, Andrew Butler, Adam Roberts y Sherryl Vint. Nueva York: Routledge.

SEED, David

2011 Science Fiction: A very short introduction. Nueva York: Oxford University Press.

SkUlt, Petter

2012 "The Post-Apocaliptic Chronotope". Consultado: 16 de marzo de 2018. <http://citeseerx.ist.psu.edu/viewdoc/downlo $\mathrm{ad}$ ?doi=10.1.1.470.6052\&rep=rep1\&type $=$ pdf $>$

Suvin, Darko

[1979] 2016 Metamorphoses of Science Fiction: On the Poetics and History of a Literary Genre. Berna: Peter Lang. Edición de Kindle.

Suvin, Darko

2008 "Of Starship Troopers and Refuseniks: War and militarism in U.S. Science fiction, Part 1 (1945-1974: Fordism)”. En New Boundaries in Political Science Fiction. Ed., Donald M. Hassler y Clyde Wilcox. Columbia: University of South Carolina Press, 115-144.

White, Michael

2005 Isaac Asimov. A life of the grand master of science fiction. Nueva York: Carroll \& Graf Publishers.

Wolfe, Gary K.

1986 Critical Terms for Science Fiction and Fantasy. A Glossary and Guide to Scholarship. Westport: Greenwood Press.

Recepción: 20/05/2018 Aceptación: 20/08/2020 